\title{
Theory of the valley-valve effect in graphene nanoribbons
}

\author{
A. R. Akhmerov, ${ }^{1}$ J. H. Bardarson, ${ }^{1}$ A. Rycerz, ${ }^{2,3}$ and C. W. J. Beenakker ${ }^{1}$ \\ ${ }^{1}$ Instituut-Lorentz, Universiteit Leiden, P.O. Box 9506, 2300 RA Leiden, The Netherlands \\ ${ }^{2}$ Institut für Theoretische Physik, Universität Regensburg, D-93040, Germany \\ ${ }^{3}$ Marian Smoluchowski Institute of Physics, Jagiellonian University, Reymonta 4, 30-059 Kraków, Poland
}

(Received 17 April 2008; published 12 May 2008)

\begin{abstract}
A potential step in a graphene nanoribbon with zigzag edges is shown to be an intrinsic source of intervalley scattering - no matter how smooth the step is on the scale of the lattice constant $a$. The valleys are coupled by a pair of localized states at the opposite edges, which act as an attractor and/or repellor for the edge states propagating in valley $K / K^{\prime}$. The relative displacement $\Delta$ along the ribbon of the localized states determines the conductance $G$. Our result $G=\left(e^{2} / h\right)[1-\cos (N \pi+2 \pi \Delta / 3 a)]$ explains why the "valley-valve" effect (the blocking of the current by a $p-n$ junction) depends on the parity of the number $N$ of carbon atoms across the ribbon.
\end{abstract}

DOI: 10.1103/PhysRevB.77.205416 PACS number(s): 73.20.Fz, 73.23.-b, 73.40.Gk, 73.63.Nm

\section{INTRODUCTION}

The massless conduction electrons in a two-dimensional carbon lattice differently respond to an electric field than ordinary massive electrons do. Because the magnitude $v$ of the velocity of a massless particle is independent of its energy, a massless electron moving along the field lines cannot be backscattered-since that would require $v=0$ at the turning point. The absence of backscattering was discovered in carbon nanotubes, ${ }^{1}$ wherein it is responsible for the high conductivity in the presence of disorder.

A graphene nanoribbon is essentially a carbon nanotube that is cut open along the axis and flattened. One distinguishes armchair and zigzag nanotubes, depending on whether the cut runs parallel or perpendicular to the carboncarbon bonds. The edges of the nanoribbon fundamentally modify the ability of an electric field to backscatter electrons. As discovered in computer simulations by Wakabayashi and Aoki, ${ }^{2}$ a potential step in a zigzag nanoribbon blocks the current when it crosses the Fermi level, forming a $p$ - $n$ junction (a junction of states in the conduction and valence bands). The current blocking was interpreted in Ref. 3 by an analogy to the spin-valve effect in ferromagnetic junctions. ${ }^{4}$ In this analogy, the valley polarization in a zigzag nanoribbon plays the role of the spin polarization in a ferromagnethence, the name "valley-valve" effect.

It is the purpose of this paper to present a theory for this unusual phenomenon. A theory is urgently needed because the analogy between a spin valve and a valley valve dramatically fails to explain the computer simulations in Fig. 1. The current blocking by the $p$ - $n$ junction turns out to depend on the parity of the number of atom rows, $N$, in the ribbon. The current is blocked when $N$ is even (zigzag configuration), while it is not blocked when $N$ is odd (antizigzag configuration; see Fig. 2). This even-odd difference (first noticed in connection to the quantum Hall effect ${ }^{5}$ ) is puzzling since zigzag and antizigzag nanoribbons are indistinguishable at the level of the Dirac equation, ${ }^{6,7}$ which is the wave equation that governs the low-energy dynamics in graphene.

\section{BREAKDOWN OF THE DIRAC EQUATION AT A POTENTIAL STEP}

The applicability of the Dirac equation rests on the assumption that a smooth potential step causes no intervalley scattering. As we now show, it is this assumption that fails in the $p-n$ junction, breaking the analogy between a spin valve and a valley valve. In a spin valve, a spin-up electron that is incident on a ferromagnetic junction, which only transmits spin-down, is simply reflected as spin-up. The current blocking can therefore be understood without invoking spin-flip scattering. In a valley valve, however, an electron in the valley $K$ incident on a $p$ - $n$ junction, which only transmits the valley $K^{\prime}$, cannot be reflected in the valley $K$. Both transmission and reflection require a switch of the valley from $K$ to
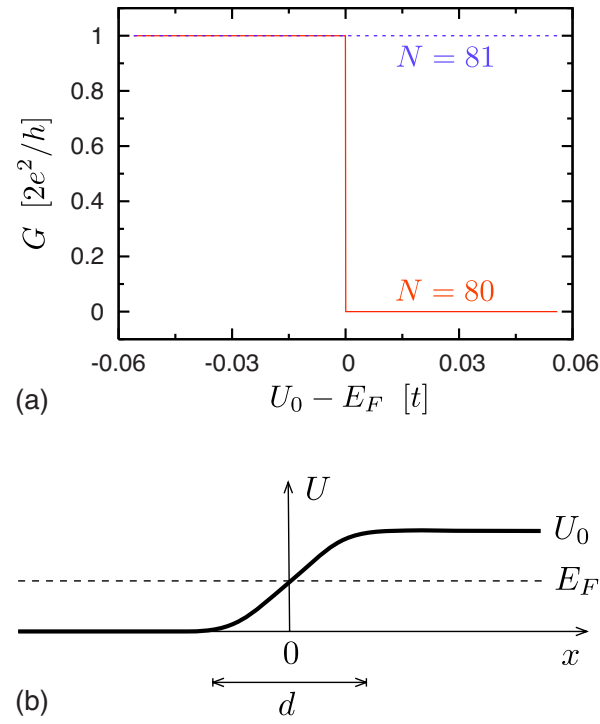

FIG. 1. (Color online) Conductance $G$ of a zigzag nanoribbon containing a potential step $U=\frac{1}{2} U_{0}[1+\tanh (2 x / d)]$. The red and blue curves are obtained by a computer simulation of the tightbinding model of graphene, with parameters $d=10 a$ and $E_{F}=0.056 t$, where $a$ is the lattice constant and $t$ is the nearestneighbor hopping energy. Upon varying $U_{0}$, the conductance abruptly switches to zero when the Fermi level $E_{F}$ is crossed and a $p$ - $n$ junction is formed (red solid curve; the deviation from an ideally quantized step function is $\$ 10^{-7}$ ). This valley-valve effect occurs only for an even number $N$ of carbon atom rows (zigzag configuration). When $N$ is odd (antizigzag configuration), the conductance remains fixed at $2 e^{2} / h$ (blue dotted curve, again quantized within $10^{-7}$ ). 


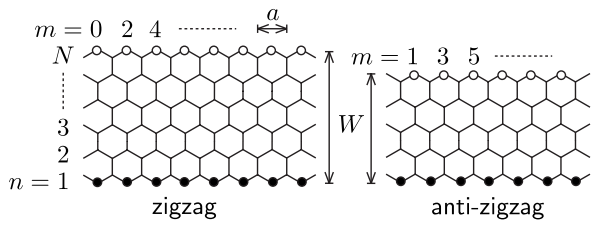

FIG. 2. Nanoribbons in the zigzag configuration (left panel, $N$ even) and in the antizigzag configuration (right panel, $N$ odd). In both cases, the atoms at opposite edges belong to different sublattices (indicated by the black and white dots).

$K^{\prime}$ (see Fig. 3). We conclude that a $p$ - $n$ junction in a zigzag nanoribbon is an intrinsic source of intervalley scattering. It does not matter how smooth the potential step might be; since the incoming and outgoing states are from different valleys, the scattering must switch valleys to preserve the current.

As we have illustrated in Fig. 3, the source of intervalley scattering is a pair of localized edge states at the $p$ - $n$ interface. It is well known that the lowest mode in a zigzag nanoribbon is confined near the edges. ${ }^{8}$ The transverse extension $\xi(\varepsilon) \sim W / \ln |\varepsilon W / \hbar v|$ of an edge state depends on the kinetic energy $\varepsilon=E_{F}-U$. We define the $p$ - $n$ interface as the line where $E_{F}-U(x, y)=0$. This line intersects the two edges at the points $\boldsymbol{r}_{ \pm}=\left(x_{ \pm}, y_{ \pm}\right)$, where $y_{+}=\left(\frac{3}{2} N-1\right) a / \sqrt{3} \equiv W$ and $y_{-}=0$, which are the $y$ coordinates of the row of atoms at the upper and lower edge, respectively (note that $\boldsymbol{r}_{ \pm}$is well defined also for a smooth interface). Upon approaching the $p-n$ interface, $\varepsilon$ decreases from $E_{F}$ to 0 ; hence, $\xi$ decreases from $\xi\left(E_{F}\right) \equiv \xi_{0}$ to a minimal value on the order of the lattice constant $a$. An electron incident on the $p$ - $n$ junction in the valley $K$ is therefore attracted to a pair of localized edge
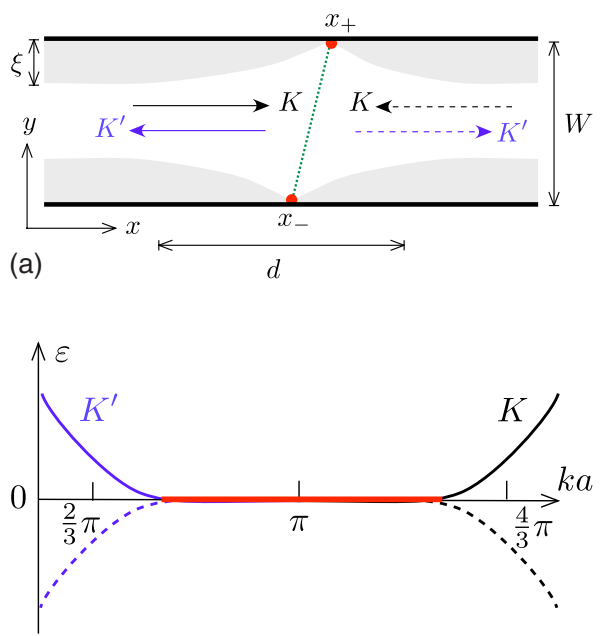

(b)

FIG. 3. (Color online) Top panel: Zigzag nanoribbon containing a $p$ - $n$ interface from $x_{-}$to $x_{+}$(dotted line). The spatial extension of the edge states in the lowest mode is indicated by the gray areas. Incoming edge states are in the valley $K$, while outgoing edge states are in the valley $K^{\prime}$. The arrows indicate the direction of propagation: in the conduction band (solid) and valence band (dashed). The corresponding dispersion relations are plotted in the lower panel. The localized (dispersionless) edge state, which is responsible for the intervalley scattering, is indicated in red. states centered at $\boldsymbol{r}_{ \pm}$. Their wave vector $k$ spans the interval of the order $1 / a$ between the valleys $K$ and $K^{\prime}$-thereby allowing for the intervalley scattering needed to repel the electron into valley $K^{\prime}$.

\section{SCATTERING THEORY BEYOND THE DIRAC EQUATION}

Now that we have identified the mechanism for intervalley scattering, we need to calculate the coupling of the propagating edge states to the pair of localized edge states in order to determine whether an incident electron is transmitted or reflected at the $p$ - $n$ interface. For this purpose, we have developed a scattering theory directly based on the tight-binding Hamiltonian

$$
H_{0}=t \sum_{\text {neighbors }}|n, m\rangle\left\langle n^{\prime}, m^{\prime}\right|,
$$

thereby going beyond the Dirac equation. The calculation is outlined below but we first present the result-which is remarkably simple: The transmission probability $T$ [and hence the conductance $\left.G=\left(2 e^{2} / h\right) T\right]$ is determined by the lateral displacement $\Delta=x_{+}-x_{-}$of the localized states, according to

$$
T=\frac{1}{2}-\frac{1}{2} \cos (N \pi+2 \pi \Delta / 3 a),
$$

for $W \gg \Delta$. This is the central result of our paper.

We have derived Eq. (2) by projecting the tight-binding Hamiltonian onto the pair of (nearly degenerate) lowest modes and then solving a scattering problem in $k$-space. As illustrated in Fig. 3 (lower panel), the incoming and outgoing states have wave vectors near $k_{\text {in }} \approx 4 \pi / 3 a$ and $k_{\text {out }} \approx 2 \pi / 3 a$, respectively. The unitary transformation of an incoming state into an outgoing state is governed by the $2 \times 2$ transfer matrix $M$ in the linear relation

$$
\Psi(k)=M\left(k, k^{\prime}\right) \Psi\left(k^{\prime}\right) .
$$

Here, we have introduced the two-component wave function $\Psi(k)=\left(\psi_{k}^{+}, \psi_{k}^{-}\right)$in $k$-space (for later use, we also introduce the Pauli matrices $\sigma_{i}$ acting on the \pm degree of freedom of the nearly degenerate lowest modes, with $\sigma_{0}$ as the $2 \times 2$ unit matrix). Once we know $M$, the scattering matrix $S=\Omega_{\text {out }} M\left(k_{\text {out }}, k_{\text {in }}\right) \Omega_{\text {in }}^{\dagger}$ follows by a change of basis such that $\Omega_{X} \Psi\left(k_{X}\right)$ (where $X$ labels "in" or "out") has the first component in the conduction band (left end of the nanoribbon) and the second component in the valence band (right end of the nanoribbon).

An analytical calculation is possible for $W \gg \xi_{0}$ when we can approximate the lowest modes $\psi_{k}^{ \pm}=2^{-1 / 2}\left(\psi_{k}^{A} \pm \psi_{k}^{B}\right)$ by 8

$$
\begin{gathered}
\psi_{k}^{A}(m, n)=C(k) e^{i m k a / 2}[-2 \cos (k a / 2)]^{n-1} \pi_{n+m+1}, \\
\psi_{k}^{B}(m, n)=C(k) e^{i m k a / 2}[-2 \cos (k a / 2)]^{N-n} \pi_{n+m},
\end{gathered}
$$

where $C(k)=\sqrt{-1-2 \cos k a}$ is a normalization factor. We have defined $\pi_{p}=1$ if $p$ is even and $\pi_{p}=0$ if $p$ is odd. The integer $n$ labels the row of atoms in the $y$ direction and $m$ labels the column of atoms in the $x$ direction (see Fig. 2). This approximation is accurate in the whole range $(2 \pi / 3 a$ 
and $4 \pi / 3 a$ ) of $k$ except within an interval of order $1 / W$ from the end points. The wave functions $\psi_{k}^{A}$ and $\psi_{k}^{B}$ are edge states, which are extended either along the lower edge (on the $A$ sublattice, indicated by black dots in Fig. 2) or along the upper edge (on the $B$ sublattice, white dots).

The nearest-neighbor tight-binding Hamiltonian [Eq. (1)] is diagonal in the basis of the modes $\psi_{k}^{ \pm}$, with matrix elements

$$
\begin{gathered}
\left\langle k, \pm\left|H_{0}\right| k^{\prime}, \pm\right\rangle= \pm \varepsilon(k) a^{-1} \delta\left(k-k^{\prime}\right), \\
\varepsilon(k)=2 t C(k)^{2}[-2 \cos (k a / 2)]^{N} .
\end{gathered}
$$

Since $\varepsilon(\pi / a-\delta k)=(-1)^{N} \varepsilon(\pi / a+\delta k)$, the parity of $N$ determines whether or not $\psi_{k}^{ \pm}$switches between the conduction and valence bands as $k$ crosses the point $\pi / a$. This band switch is at the origin of the parity dependence of the valleyvalve effect since it introduces a parity dependence on the matrices $\Omega_{\text {in }}=\sigma_{0}$ and $\Omega_{\text {out }}=\sigma_{1}^{N}$ that transforms the transfer matrix into the scattering matrix.

We model the $p$ - $n$ interface by a linear potential profile,

$$
U_{n m}=U_{x} m / 2+U_{y}(n-N / 2),
$$

which is tilted by an angle $\theta=\arctan \left(\frac{2}{3} \sqrt{3} U_{y} / U_{x}\right)$. Upon projection onto the two-component space spanned by $\Psi(k)$, the Hamiltonian $H=H_{0}+U$ becomes an integral kernel $H\left(k, k^{\prime}\right)$ with a $2 \times 2$ matrix structure:

$$
\begin{aligned}
H\left(k, k^{\prime}\right)= & \varepsilon(k) a^{-1} \delta\left(k-k^{\prime}\right) \sigma_{3}+i U_{x} a^{-2} \frac{d}{d k} \delta\left(k-k^{\prime}\right) \sigma_{0} \\
& +\frac{1}{2} N a^{-1} U_{y} \delta\left(k-k^{\prime}\right) \sigma_{1} .
\end{aligned}
$$

The integral equation

$$
a \int_{2 \pi / 3 a}^{4 \pi / 3 a} d k^{\prime} H\left(k, k^{\prime}\right) \Psi\left(k^{\prime}\right)=E \Psi(k)
$$

amounts to a system of two first-order differential equations:

$$
\left(\varepsilon(k) \sigma_{3}+i U_{x} a^{-1} \sigma_{0} \frac{d}{d k}+\frac{1}{2} N U_{y} \sigma_{1}\right) \Psi(k)=E \Psi(k) .
$$

This system directly gives an expression for the transfer matrix,

$$
\begin{gathered}
M\left(k, k^{\prime}\right)=\exp \left[i\left(k^{\prime}-k\right) a E / U_{x}\right] \mathcal{T} \exp \left[i \int_{k^{\prime}}^{k} d q \Omega(q)\right], \\
\Omega(q)=\frac{\varepsilon(q) a}{U_{x}} \sigma_{3}+\frac{\Delta}{2} \sigma_{1} .
\end{gathered}
$$

The scalar phase factor $\exp \left[i\left(k^{\prime}-k\right) a E / U_{x}\right]$ has no effect on the transmission probability so we will omit it in what follows. The symbol $\mathcal{T}$ orders the operators in the exponent with respect to the variable $q$ (from $q=k$ at the left to $q=k^{\prime}$ at the right). The scattering matrix follows from

$$
S=\sigma_{1}^{N} M\left(k_{\text {out }}, k_{\text {in }}\right) .
$$

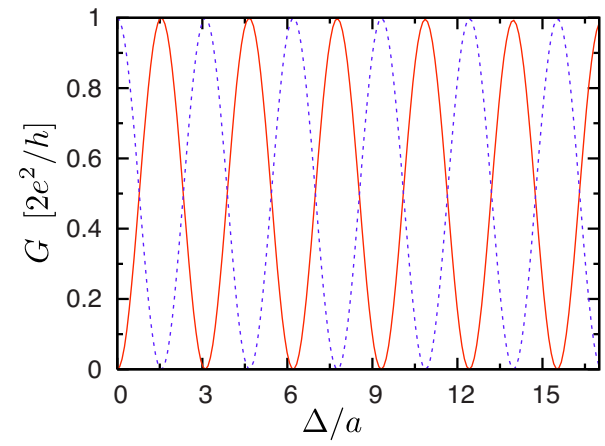

FIG. 4. (Color online) Conductance for a tilted $p$ - $n$ interface, with potential $U=\frac{1}{2} U_{0}\{1+\tanh [2(x-y \tan \theta) / d]\}$, at fixed $U_{0}=2 E_{F}$ as a function of the relative displacement $\Delta \equiv x_{+}-x_{-}=W \tan \theta$ of the intersection of the interface with the edges of the nanoribbon. The parameters are the same as those in Fig. 1, which corresponds to $\Delta=0$. The data from this computer simulation is described by the analytical result [Eq. (2)].

We may analytically evaluate Eq. (12) if $W \gg \Delta$ because then, the integration interval can be separated into subintervals in which the contribution of one of the terms can be neglected. The calculation is described in the Appendix. The result is

$$
M\left(k_{\mathrm{out}}, k_{\mathrm{in}}\right)=e^{i \alpha \sigma_{3}} \exp \left[-i(\pi \Delta / 3 a) \sigma_{1}\right] e^{i \alpha^{\prime} \sigma_{3}}
$$

with a phase shift $\alpha=(-1)^{N} \alpha^{\prime}$ that need not be determined. Substitution into Eq. (14) yields the result [Eq. (2)] for the transmission probability $T=\left|S_{12}\right|^{2}$.

The regime $\Delta \gtrsim W$ can be analyzed by a numerical evaluation of the integral [Eq. (12)]. The result, which is shown in Fig. 5 (solid curve), is that the conductance oscillations are damped for $\Delta \gtrsim W$.

\section{COMPARISON TO COMPUTER SIMULATIONS}

The current blocking $(T=0)$ that was obtained in the computer simulations of Refs. 2 and 3 is the special case that $N$ is even, $\Delta=0$, which corresponds to a zigzag configuration with a potential $U$ independent of $y$. In the antizigzag configuration ( $N$ odd), we have $T=1$ instead, which is in accord with the simulations in Fig. 1. More generally, we can tilt the interface so that $\Delta \neq 0$. The simulations for a tilted $p$ - $n$ interface shown in Fig. 4 are well described by the analytical result [Eq. (2)] for $\Delta \leqslant W \simeq 70 a$. Note in particular, the sum rule $G(N)+G(N+1) \approx e^{2} / h$ was first observed in the computer simulations in Ref. 5.

For larger $\Delta / W$, a phase shift appears and a reduction in the amplitude of the oscillations occurs with $G \simeq 0$ for $\Delta \gtrsim W$. We compare the conductance calculated by the numerical evaluation of the integral [Eq. (12)] to the data from the computer simulations and find good agreement; see Fig. 5.

\section{EXTENSIONS OF THE THEORY}

The theory presented so far can be extended in several ways. We have assumed that the width $W$ of the nanoribbon 


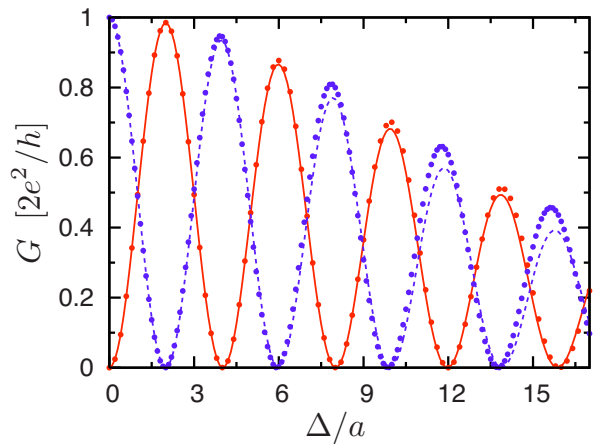

FIG. 5. (Color online) Comparison between results of computer simulations (dots) and numerical evaluation of Eq. (12). The parameters are $N=20$ (solid line) and $N=21$ (dashed line), $U_{0}=2 E_{F}=0.0058 t$, and $d \equiv E_{F} / U_{x}=100 a$.

is sufficiently narrow that there is only a single propagating mode at the Fermi level, which requires ${ }^{3}$ that $W<4 a \tau / E_{F}$. The assumption can be relaxed in the case of a smooth $p-n$ interface because higher modes have an exponentially small transmission probability if the Fermi wavelength ${ }^{9}$ $\lambda_{F} \simeq W \ll d$.

Next-nearest-neighbor hopping is not included in the theory and one might be concerned that it could substantially modify our result because the edge states are then no longer dispersionless. ${ }^{10}$ We have found that this is actually not a relevant perturbation: Next-nearest-neighbor hopping (with hopping energy $\left.t^{\prime}\right)$ adds a term $2 t^{\prime}(2+\cos k a) a^{-1} \delta\left(k-k^{\prime}\right) \sigma_{0}$ to the projected Hamiltonian [Eq. (9)]. This is an irrelevant perturbation because its only effect is to multiply the transfer matrix [Eq. (12)] by a scalar phase factor.

As a check, we have repeated the computer simulations with the inclusion of next-nearest-neighbor hopping ${ }^{11}$ in the tight-binding model (for the realistic ratio $t^{\prime} / t=0.1$ ). As shown in Fig. 6, the result [Eq. (2)] still applies for $\Delta \ll W$.

Equation (2) was derived for a linear potential profile $U$ but the derivation can be extended to include a smoothly varying potential landscape $\delta U$ (smooth on the scale of the

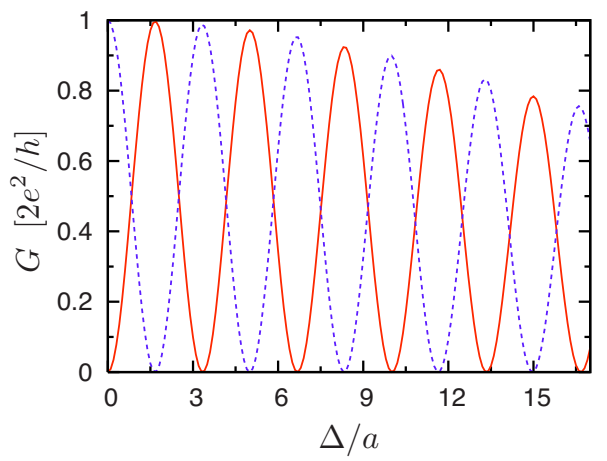

FIG. 6. (Color online) The conductance of a tilted $p-n$ interface with next-nearest neighbor hopping included $\left(t^{\prime} / t=0.1\right)$. The parameters of the ribbon are $E_{F}=0.19 t, U_{0}=0.16 t$, and $d=100 a$. The number of atoms across the ribbon is $N=40$ (solid line) and $N=41$ (dashed line). lattice constant). Electrostatic disorder therefore affects the conductance only through the lateral displacement $\Delta$ of the points on the boundary at which $U+\delta U-E_{F}=0$.

Edge disorder cannot be accounted for in this simple way but in view of the small lateral extension of the localized edge state, we might not need a well-defined zigzag edge over long distances in order for Eq. (2) to apply.

\section{CONCLUSION}

In conclusion, we have presented a theory for the current blocking by a $p-n$ junction in a zigzag nanoribbon. The dependence on the parity of the number of atoms $N$ across the ribbon, which was not noticed in earlier computer simulations, ${ }^{2,3}$ is explained in terms of the parity of the lowest mode under a switch of sublattice: The incident and transmitted modes have opposite parity for even $N$, leading to a complete reflection $(G=0)$, while they have the same parity for odd $N$, leading to a complete transmission $\left(G=2 e^{2} / h\right)$. A variation in the electrostatic potential in the direction transverse to the ribbon can invert the parity dependence of the conductance, while preserving the sum rule $G(N)+G(N+1)$ $\approx 2 e^{2} / h$.

This switching behavior may have device applications if the structure of the edges can be controlled (which is not the case in presently available samples). Even if such control is not forthcoming, the mechanism for current blocking proposed here can be operative in an uncontrolled way in disordered nanoribbons, producing highly resistive $p-n$ interfaces at random positions along the ribbon. Conduction through the resulting series of weakly coupled regions would show an activated temperature dependence as a result of the Coulomb blockade, ${ }^{12}$ as experimentally observed. ${ }^{13,14}$

\section{ACKNOWLEDGMENTS}

This research was supported by the Dutch Science Foundation NWO/FOM. A.R. acknowledges support from the Alexander von Humboldt-Stiftung, the Polish Ministry of Science (Grant No. 1-P03B-001-29), and the Polish Science Foundation (FNP). We acknowledge the help of J. Tworzydło with the computer simulations.

\section{APPENDIX: EVALUATION OF THE TRANSFER MATRIX}

To evaluate the transfer matrix $M\left(k_{\text {out }}, k_{\text {in }}\right)$ in the regime $W \gg \Delta$, we use the fact that the energy $\varepsilon(k)$ of the lowest modes exponentially decays $\sim \exp (-N a \delta k)$ away from the integration limits $k_{\text {in }}$ and $k_{\text {out }}$ [see Eq. (7)]. We separate the integration in the momentum-ordered exponent [Eq. (12)] into three intervals:

$$
\begin{aligned}
M\left(k_{\text {out }}, k_{\text {in }}\right)= & \mathcal{T} \exp \left[i \int_{k_{2}}^{k_{\text {out }}} \Omega(q) d q\right] \mathcal{T} \exp \left[i \int_{k_{1}}^{k_{2}} \Omega(q) d q\right] \\
& \times \mathcal{T} \exp \left[i \int_{k_{\text {in }}}^{k_{1}} \Omega(q) d q\right] .
\end{aligned}
$$

We choose $k_{\text {in }}-k_{1}=k_{2}-k_{\text {out }} \geq 1 / W$ such that $\left|\varepsilon\left(k_{1}\right)\right|$ $=\left|\varepsilon\left(k_{2}\right)\right| \lesssim U_{x}$. Then, the contribution of the term $\sigma_{1} \Delta / 2$ in 
$\Omega(q)$ to the integrals over the first and the third intervals is on the order of $\Delta / W \ll 1$ so that this term may be neglected. The contribution of the term $\sigma_{3} \varepsilon(q) a / U_{x}$ to the integral over the second interval is on the order of $a / W \ll 1$ so it can also be neglected. The three integrals can now be analytically evaluated, with the result:

$$
M\left(k_{\text {out }}, k_{\text {in }}\right)=e^{i \alpha \sigma_{3}} \exp \left[i\left(k_{2}-k_{1}\right) \frac{\Delta}{2} \sigma_{1}\right] e^{i \alpha^{\prime} \sigma_{3}}
$$

This is equivalent to Eq. (15) since $k_{2}-k_{1}$ $=-2 \pi / 3 a+\mathcal{O}(1 / W)$.
${ }^{1}$ T. Ando, T. Nakanishi, and R. Saito, J. Phys. Soc. Jpn. 67, 2857 (1998).

${ }^{2}$ K. Wakabayashi and T. Aoki, Int. J. Mod. Phys. B 16, 4897 (2002).

${ }^{3}$ A. Rycerz, J. Tworzydło, and C. W. J. Beenakker, Nat. Phys. 3, 172 (2007).

${ }^{4}$ S. A. Wolf, D. D. Awschalom, R. A. Buhrman, J. M. Daughton, S. von Molnar, M. L. Roukes, A. Y. Chtchelkanova, and D. M. Treger, Science 294, 1488 (2001).

${ }^{5}$ J. Tworzydło, I. Snyman, A. R. Akhmerov, and C. W. J. Beenakker, Phys. Rev. B 76, 035411 (2007).

${ }^{6}$ L. Brey and H. A. Fertig, Phys. Rev. B 73, 235411 (2006).

${ }^{7}$ The dependence of boundary conditions on the number $N$ of atoms across the ribbon is a key distinction between zigzag and armchair edges. The boundary condition of the Dirac equation for an armchair nanoribbon depends on $N$ (modulo 3), but there is no $N$ dependence for a zigzag nanoribbon.

${ }^{8}$ K. Nakada, M. Fujita, G. Dresselhaus, and M. S. Dresselhaus, Phys. Rev. B 54, 17954 (1996).
${ }^{9}$ V. V. Cheianov and V. I. Fal'ko, Phys. Rev. B 74, 041403(R) (2006).

${ }^{10}$ N. M. R. Peres, A. H. Castro Neto, and F. Guinea, Phys. Rev. B 73, 195411 (2006).

${ }^{11}$ Next-nearest-neigbor hopping gives both a displacement and a finite width to the energy interval $2 t^{\prime}<\varepsilon<3 t^{\prime}$ in which conduction and valence bands coincide [with $\varepsilon(2 \pi / 3 a)=3 t^{\prime}$ $=\varepsilon(4 \pi / 3 a)$ and $\left.\varepsilon(\pi / a)=2 t^{\prime}\right]$. To form a $p$ - $n$ junction, the Fermi level should lie above this interval in the $n$-region and below this interval in the $p$ region. Equation (2) then still holds, with $\Delta$ $=x_{+}-x_{-}$calculated from the lateral displacement of the points $\mathbf{r}_{ \pm}$on the boundary at which $U-E_{F}=3 t^{\prime}$.

${ }^{12}$ F. Sols, F. Guinea, and A. H. Castro Neto, Phys. Rev. Lett. 99, 166803 (2007).

${ }^{13}$ M. Y. Han, B. Özyilmaz, Y. Zhang, and P. Kim, Phys. Rev. Lett. 98, 206805 (2007).

${ }^{14}$ Z. Chen, Y.-M. Lin, M. J. Rooks, and Ph. Avouris, Physica E (Amsterdam) 40, 228 (2007). 